\title{
FECHAMENTO PRECOCE DAS COLOSTOMIAS EM PACIENTES COM TRAUMA DO RETO: UM ESTUDO PROSPECTIVO E CASUALIZADO
}

\author{
EARLY COLOSTOMY CLOSURE IN PATIENTS WITH RECTAL TRAUMA: \\ A PROSPECTIVE RANDOMIZED TRIAL
}

\author{
Paulo Roberto Lima Carreiro ${ }^{1}$ \\ Alcino Lázaro da Silva, TCBC-MG \\ Wilson Luiz Abrantes, TCBC-MG3
}

\begin{abstract}
RESUMO: Foi feito um estudo prospectivo e casualizado de 35 pacientes portadores de colostomias devido a lesões traumáticas do reto admitidos no Hospital Jõao XXIIII no período de novembro de 1994 a junho de 1997. O objetivo foi avaliar os resultados do fechamento precoce das colostomias nestes pacientes. Após o atendimento inicial, os pacientes foram sorteados de acordo com o número do registro de admissão em dois grupos: os do grupo $1(\mathrm{~N}=14)$ foram submetidos ao fechamento precoce da colostomia programado para o $10^{\mathrm{o}}$ dia pós-operatório do tratamento da(s) lesão(ões) e os do grupo $2(\mathrm{~N}=21)$ submetidos ao fechamento tardio da colostomia, programado para oito semanas após a operação inicial. Nos dois grupos, o restabelecimento do trânsito intestinal somente foi realizado após o fechamento da lesão retal confirmado por um estudo radiológico contrastado. Houve um predomínio de pacientes jovens, do sexo masculino e vítimas de traumatismo penetrante. Todos eram portadores de uma colostomia em alça. A taxa global de complicações após o fechamento das colostomias foi de $25,7 \%$, com a infecção de ferida operatória sendo a complicação mais freqüente $(17,1 \%)$. No grupo 1, as complicações ocorreram em 35,7\% dos casos e, no grupo 2, em 19,1\% (p= 0,423). A análise dos resultados permitiu-nos concluir que a taxa de complicações, a duração da operação para o fechamento da colostomia e o tempo total de permanência hospitalar não apresentaram diferenças significantes entre os dois grupos. Os pacientes submetidos ao fechamento precoce (grupo 1) permaneceram apenas 10 dias em média com a colostomia, enquanto nos pacientes do grupo 2 a média de permanência com a colostomia foi de 66,3 dias ( $\mathrm{p}<0,001$ - Teste de Kruskal-Wallis). Baseados nestes resultados, concluímos que os pacientes portadores de colostomias utilizadas para o tratamento de lesões traumáticas do reto e que não apresentem complicações da operação inicial, poderão ser submetidos ao seu fechamento a partir do $10^{\circ}$ DPO da operação inicial.
\end{abstract}

Descritores: Colostomias; Trauma de reto; Fechamento de colostomias.

\section{INTRODUÇÃO}

A colostomia é um princípio fundamental no tratamento das lesões de reto que poderá ser evitada apenas numa minoria de casos selecionados. ${ }^{1}$ Independente do tipo de colostomia realizada, o objetivo é a exclusão total do trânsito fecal, a fim de evitar a contaminação dos tecidos adjacentes pelo extravasamento de fezes e permitir condições locais para a cicatrização completa da lesão. O trânsito intestinal será restabelecido posteriormente.

As taxas de mortalidade e de morbidez no fechamento das colostomias variam de $0 \%$ a $4,5 \%$ e $0 \%$ a $50 \%$, respectivamente. $^{2}$ Vários estudos tentaram definir quais os fatores relacionados à mortalidade e à morbidez do fecha-

1. Pós-Graduando do Curso de Mestrado em Cirurgia da UFMG. Cirurgião dos Hospitais João XXIII, Mater Dei e Santa Casa de Belo Horizonte.

2. Professor Titular de Cirurgia do Aparelho Digestivo da UFMG.

3. Chefe da Clínica Cirúrgica do Hospital João XXIII.

Recebido em 2/12/99

Aceito para publicação em 2/5/2000

Trabalho realizado no no Hospital João XXIII e Santa Casa de Belo Horizonte-MG. 
mento das colostomias, assim como o tempo ideal para a realização desta operação. A avaliação desses resultados, entretanto, é prejudicada pelo fato de, na maioria dos estudos, predominarem pacientes com doenças inflamatórias ou neoplásicas, e não vítimas de traumas. Não foi encontrada nenhuma referência em que esse estudo tenha sido feito de maneira comparativa e casualizada. Além disso, acreditamos que os pacientes vítimas de trauma, geralmente jovens e hígidos, não necessitam permanecer um longo período de dois a três meses com uma colostomia, conforme recomendado pela maioria dos trabalhos. O objetivo da colostomia nestes pacientes é apenas a exclusão temporária do trânsito intestinal até a cicatrização da(s) lesão(ões) do reto.

Diante destes dados, o autor se propõe a estudar o fechamento precoce das colostomias em pacientes vítimas de lesões traumáticas do reto, observando as taxas de mortalidade, morbidez e permanência hospitalar, a fim de avaliar a exeqüibilidade ou não do procedimento.

\section{MÉTODO}

Pacientes portadores de lesão(ões) do reto nos quais uma colostomia foi realizada como parte do tratamento foram incluídos no estudo. Todos os pacientes foram operados no Hospital João XXIII - Fundação Hospitalar do Estado de Minas Gerais (FHEMIG), Belo Horizonte - MG, no período de novembro de 1994 a junho de 1997 e posteriormente submetidos à nova operação para reconstrução do trânsito intestinal. Os pacientes alocados para o fechamento precoce da colostomia permaneceram internados no Hospital João XXIII e submetidos à reconstrução do trânsito intestinal na mesma internação (10o DPO). Aqueles submetidos ao fechamento tardio, receberam alta hospitalar após a operação inicial e foram reinternados em caráter eletivo após oito semanas para a reconstrução do trânsito intestinal. Nestes pacientes, o fechamento das colostomias foi realizado na Santa Casa de Belo Horizonte.

Após o atendimento inicial na sala de emergência, os pacientes foram operados pelos cirurgiões de plantão para o tratamento inicial da(s) lesão(ões) de reto.

Aqueles portadores de lesão(ões) retal(ais) nos quais uma colostomia foi realizada como parte do tratamento, independente do mecanismo de lesão, da presença ou não de outros traumas associados e do tempo de evolução, foram incluídos no estudo e sorteados de acordo com o número de registro de admissão em dois grupos:

- GRUPO 1 - Os pacientes portadores de números de registro com final par, que foram submetidos ao fechamento precoce da colostomia, programado para o $10^{\circ}$ dia pós-operatório do tratamento da(s) lesão(ões).

- GRUPO 2 - Os pacientes portadores de números de registro com final ímpar, que foram submetidos ao fechamento tardio da colostomia, programado com um intervalo de pelo menos oito semanas da sua realização.

Os pacientes sorteados para o fechamento precoce da colostomia e que apresentaram qualquer contra-indicação ao procedimento cirúrgico eletivo na data programada para a reconstrução do trânsito intestinal foram excluídos deste grupo e submetidos ao fechamento tardio da colostomia, consequientemente passando para o grupo 2 .

Nos pacientes dos dois grupos, foi realizado um estudo radiológico pré-operatório com contraste iodado hidrossolúvel na luz retal introduzido na boca distal da colostomia.

A operação para reconstrução do trânsito intestinal nos dois grupos somente foi realizada após a confirmação do fechamento da lesão retal, através do controle radiológico. No grupo 1, o estudo radiológico foi realizado no 9o dia pós-operatório (DPO) da realização da colostomia, e o fechamento da mesma no dia seguinte (10 DPO ). Os pacientes do grupo 2, após a recuperação da operação inicial, receberam alta hospitalar e, após um intervalo de oito semanas, foram submetidos ao controle radiológico em nível ambulatorial. Estes pacientes foram internados posteriormente, e o fechamento da colostomia realizado em caráter eletivo.

Naqueles casos em que o estudo radiológico evidenciou escape de contraste, traduzindo o não fechamento da lesão retal, os pacientes não foram submetidos à operação para reconstrução do trânsito intestinal. Nesta situação, os pacientes do grupo 1 foram excluídos deste grupo e submetidos ao fechamento tardio da colostomia, após novo estudo radiológico confirmando a oclusão da lesão. Nos pacientes do grupo 2, em que houve escape de contraste no controle radiológico, este estudo foi repetido com intervalos de sete dias, até que se confirmasse o fechamento da lesão.

O preparo intestinal pré-operatório foi o mesmo nos dois grupos e realizado da seguinte forma :

- Dieta líquida sem resíduos pelo menos 24 horas antes da operação. Jejum na véspera.

- Solução de manitol a $10 \%, 2.000 \mathrm{~cm}^{3}$ ingeridos num período aproximado de duas horas $\left(150 \mathrm{~cm}^{3}\right.$ de $10 \mathrm{em}$ $10 \mathrm{~min})$.

- Metoclopramida: uma ampola IM, uma hora antes de iniciar o manitol.

- Sulfato de Neomicina: $1,0 \mathrm{~g}$ VO de $8 / 8$ horas, 24 horas antes da operação.

- Metronidazol: $800 \mathrm{mg}$ VO de 8/8 horas, 24 horas antes da operação e $500 \mathrm{mg} \mathrm{EV}$, uma hora antes do início da operação e mantido de $8 / 8$ horas no primeiro dia pósoperatório.

- Gentamicina: 1,0 a $1,5 \mathrm{mg} / \mathrm{Kg} \mathrm{EV}$, uma hora antes do início da operação e mantido de $8 / 8 \mathrm{~h}$ no primeiro dia pós-operatório.

- Clister glicerinado a $12 \%, 1000 \mathrm{~cm}^{3}$ via retal na véspera da operação.

Todas as operações de reconstrução do trânsito intestinal foram realizadas pelo mesmo cirurgião (PRLC), a fim de se eliminarem variantes relacionadas à técnica operatória. As operações foram realizadas obedecendo às seguintes etapas:

- incisão da pele ao redor da estomia;

- dissecção para a liberação completa da alça intestinal exteriorizada dos planos subcutâneo e músculo-aponeurótico.

- desbridamento das bocas anastomóticas; 
- anastomose com pontos separados, em plano único seromuscular com fio inabsorvível monofilamentar de polipropileno $3 \times 0$

- reposicionamento do segmento intestinal na cavidade peritoneal;

- síntese por planos.

Nos casos em que foi necessária a ressecção intestinal devido ao edema da ostomia, a técnica para a realização da anastomose foi a mesma.

Foram avaliados 35 pacientes, sendo que em 40\% (14 casos) foi realizado o fechamento precoce da colostomia e nos demais $60 \%$ (21 casos) realizou-se o fechamento tardio da colostomia.

De um modo geral, observaram-se $14,3 \%$ de casos do sexo feminino e $85,7 \%$ do sexo masculino. No grupo 1, 78,6\% de casos eram do sexo masculino e $21,4 \%$ do sexo feminino. No grupo 2, 90,5\% eram pacientes do sexo masculino e 9,5\% do sexo feminino.

A idade dos pacientes variou de cinco a 54 anos, com uma média igual a 22 anos. No grupo 1, esta variação foi de cinco a 41 anos, com média igual a 22,9 anos e, no grupo 2 , de 7 a 54 anos, com média igual a 21,8 anos.

Quanto ao tipo de trauma, 60\% tiveram trauma do tipo penetrante, $11,4 \%$ do tipo contuso e $28,6 \%$ por empalamento. No grupo $1,78,6 \%$ dos pacientes tiveram um trauma do tipo penetrante, $7,1 \%$ do tipo contuso e $14,3 \%$ por empalamento. No grupo 2 , observaram-se $47,6 \%$ dos pacientes com um trauma penetrante, $14,3 \%$ com trauma contuso e $38,1 \%$ de casos com trauma por empalamento.

Com o objetivo de avaliar a gravidade das lesões apresentadas pelos pacientes e comparar os resultados entre os dois grupos foram utilizados índices de trauma (trauma scores). Esta avaliação foi feita através do Injury Severity Score-ISS, Revised Trauma Score-RTS e do TRISS ${ }^{3-5}$. O ISS é um índice baseado em parâmetros anatômicos, o RTS utiliza parâmetros fisiológicos e o TRISS é uma combinação destes dois métodos.

Nos pacientes portadores de lesão do reto extraperitoneal nos quais não foi possível visualizar a lesão nem no pré-operatório através do exame proctológico, nem durante o ato cirúrgico, estas lesões foram consideradas como grau 2, segundo a classificação OIS-Organ Injury Scale ${ }^{6}$. Para o cálculo do ISS, o valor da AIS-Abreviated Injury Scale utilizado foi $3^{6}$.

No que se refere ao escore RTS, todos os pacientes com fechamento precoce apresentaram valor igual a 7,8 e, no grupo com fechamento tardio, houve uma variação de 6,8 a 7,8, com média igual a 7,7. Para o ISS, a variação no grupo com fechamento precoce foi de nove a 25 , com média igual a 11,6 e, no grupo com fechamento tardio, este escore variou de nove a 16 , com média igual a 11,5 . No que diz respeito ao TRISS, constatou-se uma pequena variação $(0,98$ a 0,99$)$ no grupo com fechamento precoce e uma variação de 0,94 a 0,99 no grupo com fechamento tardio.

Em todos os pacientes do grupo 1, a lesão estava localizada na porção extraperitoneal do reto (EP) e não foi reparada. Em oito destes pacientes $(57,1 \%)$, as lesões não foram visualizadas, sendo o diagnóstico da lesão estabelecido pela presença de sangue no reto. Já no grupo 2, 33,3\% dos pacientes tinham lesões EP não reparadas e em três pacientes não foi possível identificar as lesões. Em 42,9\%, a lesão se localizava no reto intraperitoneal (IP), em 4,8\%, tanto no reto EP quanto no IP, e em $19,1 \%$, na porção EP, com as lesões tendo sido reparadas.

Em 33 pacientes $(94,3 \%)$, foi realizada uma sigmoideostomia em alça e em apenas dois optou-se por uma colostomia em alça no ângulo esplênico.

No grupo com fechamento precoce, todos os pacientes tiveram uma sigmoideostomia em alça e, no grupo com fechamento tardio, esta característica foi observada em $90,5 \%$ dos casos e, nos demais $(9,5 \%)$, foi realizada uma colostomia em alça no anglo esplênico.

A presença de lesões associadas foi observada em 21 pacientes $(60 \%)$, sendo a bexiga e o intestino delgado respectivamente os órgãos mais freqüentemente acometidos (Tabela 1).

Tabela 1

Incidência Global de Lesões Associadas

\begin{tabular}{l|c}
\hline Lesão & No de pacientes \\
\hline Bexiga & 10 \\
Int. delgado & 8 \\
Int. grosso & 5 \\
Partes moles & 3 \\
Estômago & 1 \\
Duodeno & 1 \\
Uretra & 1 \\
Vascular & 1 \\
Óssea & 1 \\
\hline
\end{tabular}

No grupo 1, as lesões associadas estavam presentes em sete pacientes $(50 \%)$ e, no grupo 2 , em 14 pacientes $(66,7 \%)$.

No levantamento das diferenças entre os dois grupos quanto às variáveis categóricas de interesse, utilizouse o teste do Qui-quadrado e, nos casos em que não foi possível utilizar o teste do Qui-quadrado, utilizou-se o teste exato de Fisher. Para a análise das variáveis contínuas, utilizou-se o teste de Kruskal-Wallis. Todos os resultados foram considerados significativos no nível de significância de $5 \%$ ( $\mathrm{p}<0,05)$, tendo, portanto, $95 \%$ de confiança em que os resultados estejam corretos.

\section{RESULTADOS}

Nos dois grupos predominaram pacientes jovens e do sexo masculino, não sendo observadas diferenças entre os grupos em relação a estas variáveis. 
Observou-se uma predominância global de trauma penetrante no estudo. Não foi possível avaliar se houve diferenças significantes entre os dois grupos quanto ao tipo de trauma.

Quanto aos escores de trauma (RTS, ISS e TRISS), verificou-se que os dois grupos são similares, ou seja, não se constataram diferenças significantes entre os grupos.

No grupo 1 todos os pacientes eram portadores de lesão do reto EP e no grupo 2, observou-se um predomínio de lesões do reto IP $(42,9 \%)$. Não foi possível avaliar se os dois grupos diferenciavam-se quanto a este aspecto.

As lesões associadas estavam presentes em $50 \%$ dos pacientes do grupo 1 e em $66,7 \%$ dos pacientes do grupo 2, sendo a bexiga e o intestino delgado os órgãos mais lesados. Não houve diferenças significantes entre os grupos quanto a esta característica ( $\mathrm{p}=0,324$ - Teste Qui-quadrado).

A taxa global de complicações após o fechamento da colostomia foi de $25,7 \%$ (nove pacientes). As complicações mais comuns foram as infecções de ferida operatória $(17,1 \%)$. Apenas dois pacientes $(2,7 \%)$ tiveram complicações relacionadas à linha de sutura intestinal, sendo uma fístula no grupo 2 e uma estenose no grupo 1 . No grupo de pacientes com fechamento precoce, em 35,7\% dos casos houve complicações no fechamento da colostomia e no grupo com fechamento tardio elas ocorreram em 19,1\% de casos. Não houve diferenças significantes entre os dois grupos ( $\mathrm{p}=0,423$ - Teste de Fischer).

O tempo decorrido entre a primeira operação e o fechamento da colostomia variou de nove a 12 dias, com média igual a 10 dias no grupo $1 \mathrm{e}$, no grupo 2 , houve uma variação de 38 a 100 dias, com média igual a 66,3 dias, sendo que os dois grupos diferenciaram-se significativamente quanto a este aspecto ( $<<0,001$ - Teste de Kruskal Wallis).

Em relação ao tempo total de hospitalização, não houve diferenças significantes entre os grupos. Considerando-se os pacientes com fechamento precoce, o tempo de hospitalização variou de 14 a 30 dias, com média igual a 16,6 dias e, no grupo com fechamento tardio, variou de nove a 51 dias, com média igual a 17,7 dias $(\mathrm{p}=0,242$ - Teste de Kruskal Wallis).

No que se refere à duração da operação para reconstrução do trânsito intestinal, os resultados foram semelhantes nos dois grupos, não havendo diferenças significantes ( $p=0,237$ - Teste de Kruskal Wallis). No grupo 1, houve uma variação de 50 a 170 minutos, com média igual a 80,2 minutos e, no grupo 2, houve uma variação de 40 a 105 minutos, com média igual a 61,9 minutos.

\section{DISCUSSÃO}

A distribuição dos pacientes segundo a idade e o sexo seguiu o padrão epidemiológico esperado em pacientes vítimas de lesões traumáticas, com o predomínio do sexo masculino e faixa etária jovem. Esta observação pode ser explicada pelo fato de este grupo (jovens do sexo masculino) estar mais exposto aos agentes vulnerantes, principalmente às agressões por arma de fogo.

Nos dois grupos, houve predomínio de lesões provocadas por trauma penetrante com uma incidência global de $60 \%$, enquanto os traumas contusos corresponderam a $11,4 \%$ das lesões observadas. Esta distribuição está de acordo com a literatura, que mostra uma ocorrência de $80 \%$ de traumas penetrantes e $10 \%$ de traumas contusos ${ }^{7}$.

O objetivo da aplicação de índices de trauma é permitir uma comparação precisa entre os dois grupos em relação às condições clínicas dos pacientes (índices fisiológicos) e à gravidade das lesões apresentadas (índices anatômicos). Os resultados mostraram que os dois grupos são similares em todos os índices aplicados (RTS, ISS e TRISS) e, portanto, perfeitamente comparáveis para fins de estudo.

Em todos os pacientes, foi realizada uma colostomia em alça, seguindo os princípios preconizados por Turnbull $^{8}$. O objetivo da colostomia no tratamento das lesões do reto é proporcionar um desvio temporário do trânsito fecal, propiciando condições para a cicatrização da(s) lesão(ões) distais e evitando complicações relacionadas ao extravasamento de fezes para os tecidos adjacentes. Existem controvérsias na literatura acerca de qual tipo de colostomia alcançaria este objetivo. Alguns autores recomendam que, quando a derivação do trânsito intestinal é necessária, deve-se optar por uma colostomia terminal, pois acreditam que as colostomias em alça não proporcionam um desvio completo das fezes ${ }^{9,10}$. Vários outros autores, entretanto, consideram que a colostomia em alça é eficaz no desvio do trânsito intestinal e, conseqüentemente, indicada no tratamento das lesões retais ${ }^{11,12}$.

A não exclusão do trânsito intestinal observada por alguns, estaria relacionada a aspectos técnicos. É fundamental uma mobilização adequada e sem tensão do segmento a ser exteriorizado, de modo a obter um esporão proeminente situado acima do nível da pele ${ }^{7}$.

Em nosso estudo, a exclusão do trânsito intestinal foi estudada em 31 pacientes, sendo que em $29(93,5 \%)$ a colostomia em alça foi eficaz na derivação das fezes. Os dois pacientes que não tiveram o trânsito fecal totalmente excluído não apresentaram complicações relacionadas à passagem de fezes pelo reto. Não houve diferença entre os dois grupos quando comparados em relação à exclusão ou não do trânsito intestinal.

A vantagem na utilização da colostomia em alça é a maior facilidade técnica para a sua construção e também, posteriormente, no restabelecimento do trânsito fecal, uma vez que na maioria das vezes este procedimento será realizado por uma incisão local e sem a necessidade de ressecção intestinal ${ }^{13,14}$.

Devido à alta incidência de complicações observadas no fechamento de colostomias, alguns autores preconizaram o abandono do uso das colostomias no tratamento das lesões traumáticas do intestino grosso, recomendando a utilização de sutura primária ${ }^{15-18}$. Outros, entretanto, enfatizam que o uso da colostomia continua indicado no tratamento das lesões retais, com um índice de complicações aceitável ${ }^{19}$.

O fechamento de colostomias não deve ser classificado como uma operação simples, pois, na prática, pode ser tecnicamente difícil e, freqüentemente, seguida de complicações que prolongam a permanência hospitalar, podendo até mesmo levar o paciente ao óbito ${ }^{20-23}$. Na literatura, a 
taxa de complicações pós-operatórias varia de $4,9 \%$ a $50 \%$ e a mortalidade de $0 \%$ a $4,5 \%$. As complicações mais freqüentes são as infecciosas e as fístulas ${ }^{18,20,21,24,25}$. A taxa de complicações verificada em nosso estudo $(25,7 \%)$ está de acordo com a média observada na literatura. Não houve mortes em nossa série.

Vários trabalhos tentaram identificar os fatores que estariam relacionados às complicações da cirurgia para o fechamento de colostomias. Entre estes fatores, citamos: tempo decorrido entre a operação inicial e o fechamento da colostomia (DT oi-f), motivo pelo qual a colostomia foi indicada (etiologia), tipo de colostomia, localização da colostomia, técnica de fechamento, antibioticoprevenção e a experiência do cirurgião. A seguir, discutiremos cada uma destas variáveis.

\section{TEMPODECORRIDOENTRE AOPERAÇÃOINICIAL E OFECHAMENTO DA COLOSTOMIA ( $\triangle$ T OI-F)}

Muitos autores concordam em que o fechamento precoce das colostomias estaria relacionado a um aumento das complicações pós-operatórias ${ }^{26-28}$.

Knox et al concluíram que a melhor época para o fechamento das colostomias é entre dois e seis meses da operação inicial. Eles observaram que nos pacientes com DToi-f menor que três meses a taxa de complicações foi de $68 \%$ e naqueles com DToi-f maior que três as complicações ocorreram em $26 \%(\mathrm{p}<0,001)^{29}$.

Freund et al, num estudo com 114 pacientes, mostraram que aqueles com DToi-f menor que 12 semanas tiveram uma taxa de complicações de $81,4 \%$ comparada com $43,2 \%$ nos pacientes com DToi-f maior que 12 semanas $(\mathrm{p}<0,001)^{22}$. Outros autores, entretanto, concordam que o fechamento precoce das colostomias pode ser realizado com segurança, beneficiando os pacientes, pois não irão permanecer com a colostomia por um período de tempo prolonga$\mathrm{do}^{22,30,31}$. Chapius \& Killingback, após 100 fechamentos de colostomia consecutivos, obtiveram $15,9 \%$ de complicações nos pacientes com DToi-f menor que dois meses e $39,3 \%$ nos pacientes com DToi-f maior que dois meses ( $\mathrm{p}<$ $0,025)$. Neste estudo, $74 \%$ dos pacientes eram portadores de câncer ou diverticulite ${ }^{32}$. Mais recentemente, Renz et al e Velmahos et al obtiveram bons resultados com o fechamento precoce das colostomias em vítimas de trauma ${ }^{13,14}$.

Torna-se difícil, entretanto, uma comparação dos diferentes resultados encontrados na literatura por diversos fatores $^{2}$. Enquanto em alguns trabalhos predominam pacientes vítimas de trauma, na maioria das vezes jovens sem outros fatores de risco associados, em outros predominam portadores de câncer do intestino grosso ou diverticulite, geralmente idosos e com complicações da própria doença associadas. Existem diferenças também em relação ao tipo de colostomia. Alguns trabalhos avaliam o fechamento de colostomias em alça e outros de colostomias à Hartman, que são procedimentos cirúrgicos de magnitude diferente. Outro fator que dificulta a avaliação dos resultados é que alguns autores citam apenas as complicações locais (fístulas e abscessos), enquanto outros também consideram as complicações sistêmicas. Em nosso estudo, ao comparar- mos os resultados entre os dois grupos, observamos $35,7 \%$ de complicações no grupo 1 (precoce) e 19,1\% de complicações no grupo 2 (tardio). Esta diferença não teve significado estatístico, portanto, o fechamento precoce das colostomias pode ser realizado sem implicar num aumento da morbidez para os pacientes.

\section{ETIOLOGIA}

Alguns trabalhos estudaram a relação entre a doença que motivou a construção da colostomia com o índice de complicações pós-operatórias do fechamento e demonstraram que os pacientes com colostomias realizadas para o tratamento de câncer do intestino grosso ou diverticulite têm um risco muito maior de desenvolver complicações após o fechamento do que aqueles com colostomias para o tratamento de lesões traumáticas ${ }^{21,25,33}$.

Outros não evidenciaram qualquer relação entre a etiologia e o índice de complicações após o fechamento das colostomias ${ }^{24,28}$.

\section{TIPODE COLOSTOMIA}

Alguns autores acreditam que as colostomias em alça estariam relacionadas a uma menor incidência de complicações no pós-operatório do fechamento ${ }^{28,33}$. Outro fator que estaria relacionado a uma menor incidência de complicações após o fechamento das colostomias é a realização desta operação sem a necessidade de ressecção intestinal e anastomose ${ }^{24}$. Em nosso estudo, por motivos técnicos, em alguns pacientes do grupo 1 foi necessária a ressecção do segmento intestinal exteriorizado no momento da reconstrução do trânsito intestinal, entretanto, esta variável não foi avaliada.

\section{LOCAL DA COLOSTOMIA}

Alguns autores constataram que as complicações após o fechamento de colostomias foram mais freqüentes nas operações realizadas no cólon esquerdo quando comparadas ao cólon direito ou ao transverso. Uma possível explicação para estes resultados seria a consistência mais sólida das fezes no cólon esquerdo e uma menor mobilidade deste segmento comparado ao cólon transverso ${ }^{34}$.

\section{ANTIBIOTICOPREVENÇÃO}

O uso de antibióticos no pré-operatório também é um fator relacionado a um menor índice de complicações ${ }^{20,23}$. Em nosso estudo, o preparo pré-operatório de todos os pacientes foi o mesmo, utilizando limpeza mecânica do intestino associada à antibioticoterapia sistêmica.

\section{EXPERIÊNCIADOCIRURGIÃO}

Vários autores enfatizam que a experiência do cirurgião e uma técnica cirúrgica meticulosa são fatores importantes na redução de complicações ${ }^{20,21,23}$.

Aston \& Everett verificaram que nos pacientes operados pelos residentes a taxa de complicações foi de $26 \%$ e, 
naqueles operados por um cirurgião experiente, esta taxa foi de $2 \%(\mathrm{p}<0.0008)^{22}$. Em nosso estudo, todos os pacientes foram operados pelo mesmo cirugião (PRLC), com experiência prévia com a técnica.

Devido à multiplicidade de fatores envolvidos e às grandes diferenças entre as casuísticas na literatura, consideramos difícil uma definição precisa dos aspectos relacionados às complicações pós-operatórias do fechamento de colostomias. Relacionamos os seguintes fatores como associados a uma menor probabilidade de complicações:

- preparo pré-operatório adequado, com limpeza mecânica do intestino e antibioticoprevenção;

- técnica cirúrgica meticulosa;

- pacientes vítimas de trauma, jovens e sem doenças associadas;

- colostomias em alça;

- fechamento simples, sem a necessidade de ressecção intestinal.

A seleção dos pacientes para o fechamento precoce da colostomia é baseada em critérios clínicos e radiológicos. Neste estudo, oito pacientes foram transferidos do grupo 1 para o grupo 2, sendo seis por critérios clínicos (complicações pós-operatórias da cirurgia inicial e dois por critérios radiológicos (escape de contraste). A complicação pós-operatória inicial mais freqüente foi a fístula retovesical presente em três pacientes.

Em nosso trabalho, constatamos, através do estudo radiológico contrastado, que no 9o $\mathrm{DPO}, 77,8 \%$ dos pacientes estavam com a lesão retal cicatrizada, e portanto, na ausência de complicações clínicas, eram candidatos ao fechamento precoce da colostomia. Em todos os pacientes, o contraste radiológico utilizado foi à base de iodo, que além de proporcionar uma imagem radiológica de boa qualidade, é mais fácil de ser removido do intestino no preparo préoperatório do que o contraste baritado ${ }^{13}$.

Existem várias vantagens no fechamento precoce das colostomias. Se este procedimento for realizado na mesma admissão da operação inicial, evita a reinternação para a reconstrução do trânsito intestinal, podendo resultar numa redução do tempo de hospitalização e, conseqüentemente, dos custos hospitalares. Além disso, o fechamento precoce elimina os aspectos relacionados aos cuidados neces- sários com a ostomia pelo paciente, assim como as conseqüências socioeconômicas e psicológicas provocadas pela presença da colostomia ${ }^{14,31,35,36}$.

Em nosso estudo, não observamos diferenças no tempo de permanência hospitalar entre os grupos, porém devemos observar que os pacientes selecionados para o fechamento precoce permaneceram internados, aguardando a cirurgia de reconstrução do trânsito intestinal programada para o 10 DPO da cirurgia inicial. Este período de espera contribuiu para aumentar a média de permanência hospitalar deste grupo.

Existem alguns aspectos técnicos importantes em relação ao fechamento das colostomias que devem ser observados. Vários autores consideram o fechamento precoce das colostomias um procedimento cirúrgico difícil tecnicamente, e atribuem esta dificuldade técnica à reação inflamatória aguda com congestão e edema do segmento intestinal exteriorizado $^{32,37}$. Em nosso estudo, uma medida indireta utilizada para avaliar o grau de dificuldade técnica da cirurgia foi o tempo cirúrgico, uma vez que todas as cirurgias foram realizadas pelo autor. Não foram verificadas diferenças significantes entre os dois grupos quanto a este aspecto. Do ponto de vista prático, pudemos constatar, nos pacientes submetidos ao fechamento precoce (grupo 1), uma grande facilidade no tempo de dissecção e liberação do segmento intestinal exteriorizado, uma vez que este se encontrava frouxamente aderido aos planos subcutâneo e músculo-aponeurótico. Nos pacientes submetidos ao fechamento tardio (grupo 2), estas aderências eram firmes, dificultando este tempo cirúrgico, principalmente se a alça intestinal havia sido suturada à aponeurose na operação inicial.

Observamos também que, em alguns pacientes do grupo 1, o edema da alça dificultou bastante a realização da anastomose, e em alguns casos foi necessária a ressecção do segmento exteriorizado e a anastomose terminoterminal para a reconstrução do trânsito intestinal. Nos pacientes submetidos ao fechamento tardio, esta dificuldade não foi observada.

Baseados nos resultados obtidos, concluímos que, em pacientes portadores de colostomias para o tratamento de lesões traumáticas do reto, o restabelecimento do trânsito intestinal poderá ser feito com segurança a partir do $10^{\circ}$ DPO da operação inicial.

\begin{abstract}
A prospective and randomized trial involving 35 rectal trauma patients who were colostomized, as part of their surgical treatment, was undertaken at the João XXIII Hospital, between November 1994 and June 1997. The aim of this study was to evaluate the early results colostomy closure in this patient population. Rectal trauma victims were assigned for two groups, according to their hospital number. Group 1: Early colostomy closure, on the $10^{\text {th }}$ post-operative day, and Group 2, delayed closure, eight weeks after the initial operation. A contrast study of the rectum was performed in all patients prior to colostomy closure, in order to confirm the rectal healing. The majority were young male victims of penetrating trauma. All patients were submitted to a loop colostomy prior to the colostomy closure. The global complication rate after colostomy closure was $25.7 \%$. Postoperative wound infection was the most commom complication $(17.11 \%)$. Complications occurred in $35.7 \%$ of the cases in group 1 and $19.1 \%$ in group 2 $(p=0,423)$. We concluded that there was no significant difference between groups regarding complication rate, operative time for colostomy closure, and total hospital stay. Patients undergoing early closure (Group 1) stayed with a colostomy for an average of 10 days, while patients from delayed closure (Group 2)
\end{abstract}


had their colostomy for an average of 66.3 days ( $p<0.001$ - Kruskal-Wallis Test). Based on our findings, traumatic rectal injury victms who have a colostomy as part of their surgical treatment, may undergo early colostomy closure on the $10^{\text {th }}$ post-operative day, as long as no complication of the initial operation is present.

Key Words: Colostomies; Rectal injuries; Colostomy closure.

\section{REFERÊNCIAS}

1. Ivatury RR, Licata J, Gunduz Yilmaz et al. Management options in penetrating rectal injuries. Am Surg 1991; 57: 50-5.

2. Pittman DM, Smith LE. Complications of colostomy closure. Dis Colon Rectum 1985; 28: 836-43.

3. Baker SP, O’Neill B, Haddon Jr. W et al. The injury severity score: a method for describing patients with multiple injuries and evaluating emergency care. J Trauma 1974; 14: $187-96$

4. Champion HR, Sacco WJ, Copes WS et al. A revision of the trauma score. J Trauma 1989; 29: 623-9.

5. Boyd CR, Tolson MA, Copes WS. Evaluating trauma care: The TRISS method. J Trauma 1987; 27: 370-8.

6. Moore EE, Cogbill TH, Malangoni MA et al. Organ injury scale. Surg Clin North Am 1995; 75: 293-303.

7. Burch JM, Feliciano DV, Mattox KL. Colostomy and drainage for civilian rectal injuries: is that all? Ann Surg 1989; 209: 600-11.

8. Turnbull Jr RB. Intestinal stomas. Surg Clin North Am 1958; 38: 1361-72.

9. Miller RE, Sullivan FJ. Rectal wounds incurred in Vietnam. Military Med 1976; 141: 764-70.

10. Gustanson RG. Rectal injuries. Am Surg 1973; 39: 456-8.

11. Steinman E, Cunha JC, Branco PD et al. Ferimentos traumáticos do reto. Arq Gastroenterol 1990; 27: 120-5.

12. Mansur ES, Abrantes WL. Traumatismo do reto por corpo estranho: estudo de 50 casos. Rev Bras Colo-Proct 1992; 12: 51-5.

13. Renz BM, Feliciano DV, Sherman R. Same admission colostomy closure. A new approach to rectal wounds: a prospective study. Ann Surg 1993; 218: 279-93.

14. Velmahos GC, Degiannis E, Wells M et al. Early closure of colostomies in trauma patients - a prospective randomized trial. Surg 1995; 118: 815-20.

15. Pachter HL, Hoballah JJ, Corcoran TA et al. The morbidity and financial impact of colostomy closure in trauma patients. J Trauma 1990; 30: 1510-13.

16. Gonzales RP, Merlotti GS, Holevar MR. Colostomy in penetrating colon injury: is it necessary? J Trauma 1996; 41: 271-5.

17. Mealy K, O'Broin E, Donohve J et al. Reversible colostomy - What is the outcome? Dis Colon Rectum 1996; 39: 1227-31.

18. Berne JD, Velmahos GC, Chan LS et al. The high morbidity of colostomy closure after trauma: further support for the primary repair of colon injuries. Surg 1998; 123: 157-64.

19. Yajko RD, Norton LW, Bloemendal L et al. Morbidity of colostomy closure. Am J Surg 1976; 132: 304-6.

20. Barnett JE, Pheils M. Closure of colostomy. Aust N Z J Surg 1976; 46: 131-3.

21. Thal ER, Yeary EC. Morbidity of colostomy closure following colon trauma. J Trauma 1980; 20: 287-91.
22. Aston CM, Everett WG. Comparision of early and late closure of transverse loop colostomies. Ann R Coll Surg Engl 1984; 66: 331-3.

23. Demetriades D, Pezikis A, Melissas J et al. Factors influencing the morbidity of colostomy closure. Am J Surg 1988; 155: 594 -6.

24. Freund HR, Raniel J, Muggia-Sulam M. Factors affecting the morbidity of colostomy closure. Dis Colon Rectum 1982; 25: 712-5.

25. Livingston DH, Miller FB, Richardson JD: Are the risks after colostomy closure exaggerated? Am J Surg 1989; $158: 17-20$.

26. Foster ME, Leaper DJ, Willianson RCN et al. Changing patterns in colostomy closure : The Bristol experience 1975 - 1982. Br J Surg 1985; 72: 142-5.

27. Salley RK, Bucher RM, Rodning CB. Colostomy closure. Morbidity reduction employing a semi - standardized protocol. Dis Colon Rectum 1983; 26: 319-22.

28. Parks SE, Hastings PR. Complications of colostomy closure. Am J Surg 1985; 149: 672-5.

29. Knox AJS, Birkett FDH, Collins CD. Closure of colostomy. Br J Surg 1971; 58: 669-72.

30. Lewis A, Weeden D. Early closure of transverse loop colostomies. Annals R Coll Surg Engl 1982; 64: 57-8.

31. Geoghegan JG, Rosemberg IL. Experience with early anastomosis after the Hartmann procedure. Ann R Coll Surg Engl 1991; 73: 80-2.

32. Chapius P, Killingback M. Colostomy closure: Technique and morbidity. Aust N Z J Surg 1979; 49 : 363-8.

33. Varnell J, Pemberton LB. Risk factors in colostomy closure. Surg 1981; 89: 683-6.

34. Finch DRA. The results of colostomy closure. Br J Surg 1976; 65: 397-9.

35. White CA, Hunt JC. Psychological factors in postoperative adjustament to stoma surgery. Ann R Coll Surg Engl 1997; 79: 3-7.

36. Orbach CE, Tallent N. Modification of perceived body and of body concepts. Arch Gen Psychiat 1965; 12: 12634.

37. Wheller MH, Barker J. Closure of colostomy - a safe procedure? Dis Colon Rectum 1977; 20: 29-32.

Endereço para correspondência:

Dr. Paulo Roberto Lima Carreiro

Rua Groelândia, 422/1.102

30320-060 - Belo Horizonte-MG 